\title{
Founder mutation
}

National Cancer Institute ( $\mathrm{NCl})$

\section{Source}

National Cancer Institute (NCI). Founder mutation.

A genetic alteration observed with high frequency in a group that is or was geographically or culturally isolated, in which one or more of the ancestors was a carrier of the altered gene. This phenomenon is often called a founder effect. Also called founder variant. 